\title{
Hamiltonian Structures and Stability for Rigid Bodies with Flexible Attachments
}

\author{
P. S. Krishnaprasad \& J. E. Marsden
}

Communicated by M. GoluBITsKy

\begin{abstract}
The dynamics of a rigid body with flexible attachments is studied. A general framework for problems of this type is established in the context of Poisson manifolds and reduction. A simple model for a rigid body with an attached linear extensible shear beam is worked out for illustration. Second, the Energy-Casimir method for proving nonlinear stability is recalled and specific stability criteria for our model example are worked out. The Poisson structure and stability results take into account vibrations of the string, rotations of the rigid body, their coupling at the point of attachment, and centrifugal and Coriolis forces.
\end{abstract}

\section{Introdaction}

Recently there has been renewed interest in Hamiltonian structures and their application to problems of stability. The main original work is due to ARNOLD [1966a, b]. This has been revived and applied to a number of fluid and plasma problems by Holmes, Marsden, Ramu and Weinstein [1984, 1985] and other authors cited by them. They coined the phrase "the Energy-Casimir method" for the basic procedure. These and related methods were applied to the control and stability of dual spin spacecraft by KrishNAPRASAD [1984]. Here we put together a continuum model for flexible structures with the finite-dimensional rigid body model and use the general Hamiltonian methods to analyze nonlinear stability.

The motivating physical situation is the stability of spacecraft with attached fiexible antennas or solar panels. There is a substantial literature in the field of aerospace engineering which is devoted to problems concerning the control and stability of rigid spacecraft with fiexible components. The papers by Likins [1974], KANe \& Levinson [1980], Metrovitch \& J. N. Juang [1974], and the recent book of KANE, LIKINS \& LEVINSON [1983] should provide a useful sample. In those works, a variety of finite-dimensional approximations are used and rough 
stability criteria are presented. There are many related problems studied in the literature such as the dynamics and buckling of rotating beams and rods; see, for example, ANTMAN \& Nachman [1980], Nactaman [1985] and references therein. However, a fundamental study of Hamiltonian structures and their application to the dynamics of rigid bodies with elastic attachments presents essentially unexplored territory. Efforts in this direction may be found in MerRovrtch [1974] and in BAILliful \& Levi [1983, 1984]. The purpose of this work is to continue such investigations by including symmetry and reduction for Hamiltonian systems applied to question concerning stability of coupled rigid-elastic structures. We note that concrete stability criteria are useful in specific satellite design problems such as the Dynamics Explorer A carrying a plasma wave instrument (including a pair of 100 meter wire antennas). See HUBERT [1981].

In this paper, we explicate the Hamiltonian structures needed and derive explicit stability criteria for a simple model. We have chosen a specific linear second order shear beam (string-like) model for purposes of illustrating the method; it is not intended to be realistic. However, the procedures used are general and can be adapted to situations of interest at hand. In following papers we plan to discuss the effect of including damping and shall use a nonlinear beam model of Kirchhoff-Love type but including shear and torsion (see REISSNER [1973, 1981], Antman [1974], ANTman \& KenNy [1981] and Simo [1985]). We also plan to discuss a similar model for plates and shells.

The contents of the paper are as follows. Section 2 gives some general results concerning the reduction of Poisson manifolds that are motivated by and needed in our example. Section 3 studies the Hamiltonian structure of the example. Section 4 reviews the Energy-Casimir method and Section 5 applies it to get specific stability criteria for our example.

\section{Generallities on Poisson Structures}

We now derive some general formulas for Poisson structures on certain spaces. The idea as far as rigid bodies are concerned is to represent the flexible structure in coordinates attached to the body. Doing so introduces Coriolis and centrifugal forces which must be taken into account in a systematic way. We do this using the general theory of reduction (Marsden \& Weinstein [1974]). Reduction involves taking the quotient by a group action; this procedure can be viewed as the passage from the kinematic description of the bodies' motion relative to an inertial frame to the description relative to a non-inertial body frame. (In corresponding fiuid and plasma problems it represents the passage from Lagrangian, or material, "coordinates" to Eulerian, or spatial, "coordinates".)

The material below assumes the reader is familiar with some general theory of Poisson manifolds, reduction and the Lie-Poisson bracket on duals of Lie algebras. Expositions of this theory may be found in MArspen e others [1983] and KRISHNAPRASAD [1985]. We remark in passing that a number of the developments here parallel tbe development and applications of the theory of reduction for principal bundles (see Montgomiery, Marsden \& RATtu [1984] and Lew1S, Marspen, 
MONTGOMery \& RATu [1986]) and the theory of Poisson reduction in general (MARSDEN \& RATIU [1985]).

We begin by recalling a few facts about Lie-Poisson brackets. Let $G$ be a Lie group (finite dimensional in this paper). (f) its Lie algebra and (s) the dual space. For a (smooth) function $F: \mathscr{S}^{*} \rightarrow \mathbf{R}$, define its functional derivative $\delta F / \delta \mu$ : (B) $\rightarrow$ BS by

$$
D F(\mu) \cdot \delta \mu=\left\langle\frac{\delta F}{\delta \mu}, \delta \mu\right\rangle
$$

where $D F(\mu)$ is the (F réchet) derivative of $F, \delta \mu \in B *, \delta F / \delta \mu$ stands for $\delta F / \delta \mu$ evaluated at $\mu \in B^{*}$ and $\left.\zeta_{0}\right\rangle$ is the pairing between $B$ and $B^{*}$. We let $B^{*}$. denote (B) with the \pm Lie-Poisson bracket, which is defined by

$$
\{F, G\}_{ \pm}(\mu)= \pm\left\langle\mu,\left[\frac{\delta F}{\delta \mu}, \frac{\delta G}{\delta \mu}\right]\right\rangle,
$$

where [, ] is the Lie algebra bracket on $B$. The map from $T^{*} G$ to $G^{*}-$ given by sending $\alpha_{8} \in T_{8}^{*} G$ to $T L_{z}^{*} \cdot \alpha_{z} \in S^{*}$ is a Poisson map (in fact a momentum map) which induces a Poisson diffeomorphism of the quotient space $T^{*} G / G$ with $\mathbb{S}^{*}$. Here $L_{g}$ is left translation by $g, T L_{z}$ is its tangent, and $T L_{z}^{*}$ is the dual of $T L_{g}$. If, instead, $\alpha_{q}$ is sent to $T R_{g}^{*} \cdot \alpha_{e}$, then we get a Poisson map from $T^{*} G$ to $G^{*}$.

The quotient space $T^{*} G / G$ is called the reduction of $T^{*} G$ by $G$. Thus, left translation to the identity identifies this reduced space with the Poisson manifold $B^{*}$. For the rigid body, one takes $G=S O(3)$ and uses left translation; the reduced space is $s o(3)^{*}=\mathbf{R}^{3 *}$ and is identified with the space of angular momenta $m$ in body representation. The Lie-Poisson bracket in this case is then given on functions of $\boldsymbol{m}$ by the triple product

$$
\{F, G\}(m)=-m \cdot(\nabla F \times \nabla G) .
$$

The Euler equations for a free rigid body then are equivalent to Hamilton's equations in Poisson bracket form

$$
\dot{F}=\{F, H\} \text {, }
$$

where $H$ is the rigid body Hamiltonian. (See also Holmes \& MArsden [1983] for an exposition of this theory and its application to the heavy top.)

For a rigid body with attachments, we generalize the above scheme by replacing $T^{*} G$ by $T^{*} G \times P$ where $P$ is the phase space for the attachment. Since the body and attachment can be simultaneously rotated, we assume that $G$ acts on $P$.

Abstractly, we assume $G$ is a Lie group acting by canonical (Poisson) transformations on a Poisson manifold $P$. Define $\phi: T^{*} G \times P \rightarrow D^{*} \times P$ by

$$
\phi\left(\alpha_{z}, x\right)=\left(T L_{z}^{*} \cdot \alpha_{r} g^{-1} \cdot x\right)
$$

where $g^{-1} \cdot x$ denotes the action of $g^{-1}$ on $x \in P$. For our example, $G=S O(3)$ and $\alpha_{g}$ is a momentum variable which is given in coordinates on $T^{*} S O(3)$ by the momentum variables $p_{\phi}, p_{\theta}, p_{\varphi}$ conjugate to the Euler angles $\phi . \theta, \psi$. The mapping $\phi$ in (2.5) transforms $\alpha_{q}$ to body representation and transforms $x \in P$ to $g^{-1} \cdot x$, which represents $x$ relative to the body. 
For $\xi \in$ we let $\xi_{P}$ denote its infinitesimal generator on $P$, so $\xi_{P}$ is the veztor field on $P$ given by

$$
\xi_{P}(x)=\left.\frac{d}{d t}(\exp (t \xi) \cdot x)\right|_{t=0} .
$$

For $F, G: G * \times P \rightarrow \mathbf{R}$, let $\{F, G\}_{-}$stand for the minus Lie-Poisson bracket given by (2.2) holding the $P$ variable fixed and let $\{F, G\}_{P}$ stand for the Poisson bracket on $P$ with the variable $\mu \in B^{*}$ held fixed.

Endow ${ }^{*} \times P$ with the following bracket:

$$
\{F, G\}=\{F, G\}_{-}+\{F, G\}_{P}-d_{x} F \cdot\left(\frac{\delta G}{\delta \mu}\right)_{P}+d_{x} G \cdot\left(\frac{\delta F}{\delta \mu}\right)_{P}
$$

where $d_{x} F$ means the differential of $F$ with respect to $x \in P$ and the evaluation point $(\mu, x)$ has been suppressed.

2.1 Proposition. The bracket (2.6) makes ${ }^{*} \times P$ into a Poisson manifold and $\phi: T^{*} G \times P \rightarrow G^{*} \times P$ is a Poisson map, where the Poisson structure on $T^{*} G \times P$ is given by the sum of the canonical bracket on $T^{*} G$ and the bracket on $P$. Moreoter, $\phi$ is $G$ invariant and induces a Poisson diffeomorphism of $\left(T^{*} G \times P\right) / G$ with $G^{*} \times P$.

Proof. For $F, G: G * \times P \rightarrow R$, let $\bar{F}=F \circ \phi$ and $\bar{G}=G \circ \phi$. Then we want to show that $\{\bar{F}, \bar{G}\}_{r \cdot G}+\{\bar{F}, \bar{G}\}_{P}=\{F, G\} \circ \phi$. This will show $\phi$ is canonical. Since it is easy to check that $\phi$ is $G$ invariant and gives a diffeomorphism of $\left(T^{*} G \times P\right) / G$ with $B^{*} \times P$, it follows that $(2.6)$ represents the reduced bracket and so defines a Poisson structure.

To prove our claim, write $\phi=\phi_{G} \times \phi_{p}$. Since $\phi_{G}$ does not depend on $x$ and the group action is assumed canonical, $\{\bar{F}, \bar{G}\}_{P}=\{F, G\}_{P} \circ \phi$. For the $T^{*} G$ bracket, note that since $\phi_{G}$ is a Poisson map of $T^{*} G$ to $\sigma^{*}$, the terms involving $\phi_{G}$ will be $\{F, G\}_{-} \circ \phi$. The terms involving $\phi_{P}\left(\alpha_{r}, x\right)=g^{-1} \cdot x$ are given most easily by noting that the bracket of a function $K$ of $g$ with a function $L$ of $\alpha_{z}$ is

$$
\bar{d}_{s} K \cdot \frac{\delta L}{\delta a_{z}}
$$

where $\delta L / \delta a_{z}$ means the fiber derivative of $L$ regarded as a vector at $g$. This is paired with the covector $d_{g} K$. Letting $\Psi_{x}(g)=g^{-1} \cdot x$, we find by use of the chain rule that missing terms in the bracket are

$$
d_{x} F \cdot T \Psi_{x} \cdot \frac{8 G}{\delta \mu}-d_{x} G \cdot T \Psi_{x} \cdot \frac{\delta F}{\delta \mu} .
$$

However, $T \Psi_{x} \cdot \frac{\delta G}{\delta \mu}=-\left(\frac{\delta G}{\delta \mu}\right)_{P} \cdot \Psi_{x}$, so the preceding expression reduces to the last two terms in equation (2.6).

Before applying this to our example, it will be convenient to develop the theory a little further. Suppose that the action of $G$ on $P$ has an $A d^{*}$ equivariant momentum 
map $J: P \rightarrow G^{*}$. Consider the map $\alpha: G^{*} \times P \rightarrow G^{*} \times P$ given by

$$
\alpha(\mu, x)=(\mu+J(x), x) .
$$

Let the bracket $\{,\}_{0}$ on $\sigma^{*} \times P$ be defined by

$$
\{F, G\}_{0}=\{F, G\}_{-}+\{F, G\}_{P}
$$

Thus $\{.\}_{0}$ is $(2.6)$ with the coupling or interaction terms dropped. We claim that the map $\propto$ eliminates the coupling:

2.2. Proposition. $\alpha:\left(\Theta^{*} \times P,\{\},\right) \rightarrow\left(\mathbb{G}^{*} \times P,\{,\}_{0}\right)$ is a Poisson diffeomorphism.

Proof. For $F, G: G^{*} \times P \rightarrow \mathbf{R}$, let $\hat{F}=F \circ \alpha$ and $\hat{G}=G \circ \alpha$. Then letting $v=\mu+J(x)$, and dropping evaluation points, we conclude that

$$
\frac{\delta \hat{F}}{\delta \mu}=\frac{\delta F}{\delta y} \quad \text { and } \quad d_{x} \hat{F}=\left\langle\frac{\delta F}{\delta v}, d_{x} J\right\rangle+d_{x} F .
$$

Substituting into the bracket (2.6), we get

$$
\begin{aligned}
\{\hat{F}, \hat{G}\}= & \left.-\left\langle\mu,\left[\frac{\delta F}{\delta v}, \frac{\delta G}{\delta v}\right]\right\rangle+\{F, G\}_{P}+\left\langle\frac{\delta F}{\delta v}, d_{x} J\right\rangle,\left\langle\frac{\delta G}{\delta v}, d_{x} J\right\rangle\right\}_{P} \\
& +\left\langle\left\langle\frac{\delta F}{\delta v}, d_{x} J\right\rangle, d_{x} G\right\}_{P}+\left\{d_{x} F,\left\langle\frac{\delta G}{\delta v}, d_{x} J\right\rangle\right\}_{P} \\
& -\left\langle\frac{\delta F}{\delta v}, d_{x} J \cdot\left(\frac{\delta G}{\delta v}\right)_{P}\right\rangle-d_{x} F \cdot\left(\frac{\delta G}{\delta v}\right)_{P} \\
& +\left\langle\frac{\delta G}{\delta v}, d_{x} J \cdot\left(\frac{\delta F}{\delta v}\right)_{P}\right\rangle+d_{x} G \cdot\left(\frac{\delta F}{\delta v}\right)_{P} .
\end{aligned}
$$

However, $\left\{d_{x} F,\left\langle\frac{\delta G}{\delta v}, d_{x} J\right\rangle\right\}_{P}$ means the pairing of $d_{x} F$ with the Hamiltonian vector field associated with the one form $\left\langle\frac{\delta G}{\delta v}, d_{x} J\right\rangle$, which is $\left(\frac{\delta G}{\delta v}\right)_{P}$, by definition of the momentum map. Thus the corresponding four terms in (2.9) cancel. Let us consider the remaining terms. First of all, we consider

$$
\left\{\left\langle\frac{\delta F}{\delta v}, d_{x} J\right\rangle,\left\langle\frac{\delta G}{\delta v}, d_{x} J\right\rangle\right\}_{P}
$$

Since $J$ is equivariant, it is a Poisson map to $G_{+}^{*}$. Thus, (2.10) becomes $\left\langle J,\left[\frac{\delta F}{\delta v}, \frac{\delta G}{\delta v}\right]\right\rangle$. Similarly each of the terms $-\left\langle\frac{\delta F}{\delta v}, d_{x} J \cdot\left(\frac{\delta G}{\delta v}\right)_{P}\right\rangle$ and $\left\langle\frac{\delta G}{\delta v}, d_{x} J \cdot\left(\frac{\delta F}{\delta v}\right)_{p}\right\rangle$ equals $-\left\langle J,\left[\frac{\delta F}{\delta v}, \frac{\delta G}{\delta v}\right]\right\rangle$, so these three terms collapse to $-\left\langle J,\left[\frac{\delta F}{\delta v}, \frac{\delta G}{\delta v}\right]\right\rangle$ which combines with $-\left\langle\mu,\left[\frac{\delta F}{\delta v}, \frac{\delta G}{\delta v}\right]\right\rangle$ to produce $-\left\langle\nu,\left[\frac{\delta F}{\delta v}, \frac{\delta G}{\delta v}\right]\right\rangle=\{F, G\}_{\text {. }}$. Thus, (2.9) collapses to (2.8). 
Remark. This result is analogous to the isomorphism between the "Sternberg" and "Weinstein" representations of a reduced principal bundle. (See GunLEmin . \& STERNBERG [1984], MONTGOMIERY, MARSDEN \& RATUU [1984] and references therein.)

Recall that a Casimir function is a function whose Poisson bracket with any other function is zero. From Proposition 2.2, we get

2.3 Corollary. Suppose $C(v)$ is a Casinir function on ${ }^{*}$. Then

$$
C(\mu, x)=C(\mu+J(x))
$$

is a Casimir function on $\left(3^{*} \times P\right.$ for the bracket (2.6).

We conclude this section with some consequences of Proposition 2.1. The first is a connection with semi-direct products. Namely, we notice that if $\$$ is another Lie algebra and $G$ acts on $\$$, we can reduce $T^{*} G \times G^{*}$ by $G$.

2.4 Corollary. Giving $T^{*} G \times \Phi^{*}$ the sum of the canonical and the "." Lie-Poisson structure on $\S^{*}$, the reduced space $\left(T^{*} G \times \Phi^{*}\right) / G$ is $\mathbb{G}^{*} \times \mathscr{G}^{*}$ with the bracket

$$
\{F, G\}=\{F, G\}_{\delta^{*}}+\{F, G\}_{\delta^{*}}-d_{\nu} F \cdot\left(\frac{\delta G}{\delta \mu}\right)_{0^{*}}+d_{r} G \cdot\left(\frac{\delta F}{\delta \mu}\right)_{0^{*}}
$$

where $(\mu, v) \in \sigma^{*} \times \Phi^{*}$, which is the Lie-Poisson bracket for the semidirect product $\mathbb{B}(\mathbf{O}$.

This is compatible with, and reproduces some of the reduction results of Marsden \& others [1983], and Marsden, RATIU \& Weinstain [1984a, b] (see also HOLM, KUPERSHMIDT \& LEVERMORE [1983]). Of course such structures are important for examples like a rigid body with a fixed point in the presence of a gravitational field (see HolMEs \& MARSDEN [1983]).

Here is another result similar to Proposition 2.1 which reproduces the symplectic form on $T^{*} G$ written in body coordinates (ABRAHAM \& MARSDEN [1978, p. 315]). We phrase the result in terms of brackets.

2.5 Corollary. The map of $T^{*} G$ to (f) $^{*} \times G$ given by $\alpha_{g} \mapsto\left(T L_{z}^{*} \alpha_{r}, g\right)$ maps the canonical bracket to the following bracket on $5^{*} \times G$ :

$$
\{F, G\}=\{F, G\}_{-}+d_{s} F \cdot T L_{z} \frac{\delta G}{\delta \mu}-d_{z} G \cdot T L_{z} \frac{\delta F}{\delta \mu}
$$

where $\mu \in B^{*}$ and $g \in G$.

Proof. This is proved by the same method as in Proposition 2.1. For $F: C \times G$ $\rightarrow R$, let $\bar{F}\left(a_{g}\right)=G(\mu, g)$ where $\mu=T L * \alpha_{g}$. The canonical bracket of $\bar{F}$ and $\bar{G}$ will give the $(-)$ Lie-Poisson structure via the $\mu$ dependence. The remaining terms are

$$
\left\langle d_{p} \bar{F}, \frac{\delta \bar{G}}{\delta p}\right\rangle-\left\langle d_{z} \bar{G}, \frac{\delta \bar{F}}{\delta p}\right\rangle
$$


where $\frac{\delta \bar{F}}{\delta p}$ means the fiber derivative of $\bar{F}$ regarded as a vector field and $d_{g} \bar{G}$ means the derivative holding $\mu$ fixed. Using the chain rule, one gets (2.12).

We now combine this corollary with Proposition 2.1 to produce a Poisson structure on $\mathrm{BS}^{*} \times \mathrm{BS}^{*} \times G$. This structure may be relevant to the motion of two rigid bodies coupled with a ball and socket joint. (The exploration of this point is planned for another publication.)

2.6 Corollary. The reduced space $\left(T^{*} G \times T^{*} G\right) / G$ is identifiable with $B^{*} \times B^{*} \times G$, with the Poisson bracket

$$
\begin{aligned}
\{F, G\}\left(\mu_{1}, \mu_{2}, g\right)= & \{F, G\}_{\mu_{1}}^{-}+\{F, G\}_{\mu_{2}}^{-}-d_{z} F \cdot T R_{2} \cdot \frac{\delta G}{\delta \mu_{1}}+d_{s} G \cdot T R_{2} \cdot \frac{\delta F}{\delta \mu_{3}} \\
& +d_{2} F \cdot T L_{2} \cdot \frac{\delta G}{\delta \mu_{2}}-d_{2} G \cdot T L_{2} \cdot \frac{\delta F}{\delta \mu_{2}}
\end{aligned}
$$

where $\{F, G\}_{\mu_{1}}^{-}$is the "-" Lie-Poisson bracket with respect 10 the first variable $\mu_{1}$, and similarly for $(F, G)_{\mu_{2}}^{-}$.

Proof. The isomorphism of $\left(T^{*} G \times T^{*} G\right) / G$ with $G^{*} \times G^{*} \times G$ is implemented by the map

$$
\left(a_{r} \beta_{h}\right) \mapsto\left(T L * a_{s}, T L^{*} \beta_{h}, g^{-1} h\right) .
$$

We map this in two steps. First, map

$$
T^{*} G \times T^{*} G \rightarrow T^{*} G \times B^{*} \times G
$$

by using Corollary 2.5 . Now regard $G$ as acting on $G^{*} \times G$ by left multiplication on the last factor alone. Then map $T^{*} G \times\left(B^{*} \times G\right)$ to $B^{*} \times B^{*} \times G$ by Proposition 2.1. Noting that at the point $\left(\mu_{1}, \mu_{2}, g\right)$

we get (2.13).

$$
\left(\frac{\delta F}{\delta \mu_{1}}\right)_{\delta \cdot \times G}=\left(0, T R_{g} \cdot \frac{\delta F}{\delta \mu_{1}}\right)
$$

This bracket (2.13) can also be verified by a direct calculation using the map (2.14).

Finally, we remark that the theory in this section can be applied to a variety of situations besides those in this paper. For example, Alvarez-SaNCHEZ [1986] uses these ideas to obtain some of the results of KrishNaprasad [1985].

\section{A Rigid Body with a Mexible Attachment}

The influence of flexible attachments on the dynamics of an otherwise rigid spacecraft structure is of great interest to aerospace engineers. Such flexible attachments are common (e.g., solar panels, antennae, instrument booms, etc.) 
and pose problems in control and stabilization of the spacecraft. Here we take up a model problem of this type where the flexible attachment is a very simple linear elastic shear beam. A more realistic example involving a nonlinear beam model for the attachment will be the subject of a subsequent paper. We plan also to include questions about passive damping and related asymptotic stability.

In Figure 1 we show a rigid body with a fiexible attachment in a reference configuration. In the reference configuration the axis of the attachment is along the axis two of a frame attached to the rigid body. We then view a typical configuration of the attachment as being given by a smooth map,

$$
\sigma:[0, L] \rightarrow \mathbf{R}^{3} \text {. }
$$

Here $\sigma(s)$ is the Lagrangian (or material) position vector and $L$ is the length of the beam in its unstressed (natural) state.

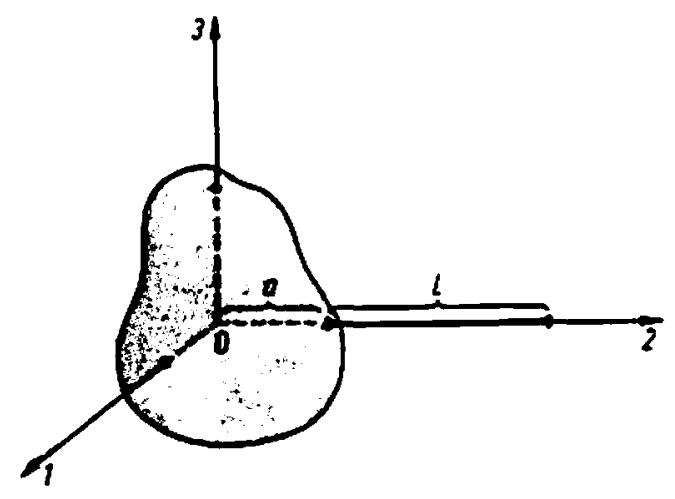

Fig. 1

Similary, $\mathcal{M}(s)$ is a Lagrangian momentum density given by $\varrho v$ (material mass per unit length) $\times$ (material velocity).

If we denote by $Q$ the space of all maps $\sigma$ then the phase space for the attachment is $P=T^{*} Q$, identified with the space of pairs $(\sigma, N)$.

The whole assemblage is moving freely in space, in the absence of external forces or torques. The center of mass of the system is inertially fixed at the origin. We now make the following (small deflection) assumption to simplify our analysis: The deflections of the attachment away from the nominal, suitably sealed by the mass of the attachment are sufficiently small that the center of mass of the rigid body has negligible motion.

Under the small deflection assumption, the configuration space of the rigid body is $S O(3)$. Furthermore, $S O(3)$ acts on (the left on) the configuration space $P$ by transporting (or swinging) the attachment around. It follows that the action of $S O(3)$ on $P$ is a Poisson action, where $P=T^{*} Q$ carries its natural (nondegenerate) Poisson structure. We are now in a position to apply Proposition 2.1 and write down the bracket $(2.6)$ on the reduced phase space so(3)* $\times P$. 
To make things more explicit, we introduce the relative position $r(s)$ and relative momentum density $M(s)$ for the attachment

$$
\begin{gathered}
r(s)=A^{-1} \sigma(s) \\
M(s)=A^{-1} M(s) .
\end{gathered}
$$

Here $A$ denotes the element of $S O(3)$ identifying the rigid body configuration. Further, let $\boldsymbol{m}$ denote the body angular momentum of the rigid body. In terms of these variables, the reduced bracket (2.6) takes the form

$$
\begin{aligned}
\{F, G\}= & -m \cdot\left(\nabla_{m} F \times \nabla G_{m}\right)+\int_{0}^{L}\left(\frac{\delta F}{\delta r} \cdot \frac{\delta G}{\delta M}-\frac{\delta F}{\delta M} \cdot \frac{\delta G}{\delta r}\right) d s \\
& +\int_{0}^{L}\left[\frac{\delta G}{\delta r}\left(\nabla_{m} F \times r\right)+\frac{\delta G}{\delta M} \cdot\left(\nabla_{m} F \times M\right)\right] d s \\
& -\int_{0}^{L}\left[\frac{\delta F}{\delta r} \cdot\left(\nabla_{m} G \times r\right)+\frac{\delta F}{\delta M} \cdot\left(\nabla_{m} G \times M\right)\right] d s .
\end{aligned}
$$

Here $F$ and $G$ are functions on the reduced (phase) space so(3)* $\times P$ which consists of the variables $(m, r, M)$.

Next, we shall apply Proposition 2.2 and its Corollary 2.3 to this situation. The action of SO(3) on $P$ has a momentum map given by the relative angular momentum of the attachment:

$$
J: P \rightarrow \operatorname{so}(3)^{*} \text { where } J(r, M)=\int_{0}^{L} r(s) \times M(s) d s .
$$

This can be checked by noting that the action of $G$ on $P$ is a cotangent lift (see Abraham \& Marsden [1978, p. 283]). Now, for any $\phi: R \rightarrow R$, the function

$$
\tilde{C}_{\triangleleft}: \operatorname{so}(3)_{-}^{*} \rightarrow \mathbf{R} \text { where } \quad \tilde{C}_{\triangleleft}(m)=\phi\left(\|m\|^{2}\right)
$$

is a Casimir function with respect to the (minus) Lie-Poisson bracket on so(3)-. From (3.4) and Corollary 2.3, we then conclude that

$$
\begin{gathered}
C_{\downarrow}: s o(3)^{*} \times P \rightarrow \mathrm{R}, \\
C_{\downarrow}(m, r, M)=\phi\left(\left\|m+\int_{0}^{2} r \times M d s\right\|^{2}\right)
\end{gathered}
$$

is a Casimir function for the Poisson bracket (3.3). A direct check of this requires the application of vector triple product identities and is left to the reader. (OF course it was doing this example by hand that prompted us to prove Proposition 2.2 and its corollary abstractly.)

We choose the Hamiltonian to be

$$
H=\frac{1}{2} \sum_{i=1}^{3} \frac{m_{i}^{2}}{I_{1}}+\frac{1}{2} \int_{0}^{L} \frac{\|M(s)\|^{2}}{e_{0}} d s+\frac{1}{2} \int_{0}^{L}\left\langle A \frac{d r}{d s}, \frac{d r}{d s}\right\rangle d s
$$


where $A$ is a positive definite symmetric matrix. Our choice of $H$ models the attachment as a "linear extensible shear beam." * Also, for the sake of simplicity, . we have dropped from $H$ a term corresponding to the kinetic energy of the attachment arising from its "swinging" along with the spinning rigid body. The coefficients $I_{1}, I_{2}, I_{3}$ represent the principal moments of inertia of the rigid body. The mass per unit length of the attachment is assumed to be a constant $\varrho_{0}$. We also impose the boundary conditions:

(point of attachment)

$$
\begin{aligned}
& r(s)=a j \text { at } s=0, \\
& r^{\prime}(s)=f \text { at } s=L .
\end{aligned}
$$

(stress free end)

Next, let us derive the equations of motion using the bracket (3.3) and Hamiltonian (3.7). The equations are determined by the requirement that

$$
\dot{F}=\{F, H\} \text {. }
$$

for all functionals $F(m, r, M)$. To unravel (3.9) to get explicit equations for $m, r$, $M$, we take the following steps.

Suppose $F=F(m)$ is a function of $m$ alone. Then

$$
\dot{F}=\nabla_{m} F \cdot \dot{m} \text {. }
$$

On the other hand, from (3.9) and formula (3.3) for the bracket,

$$
\dot{F}=-m \cdot\left(\nabla_{m} F \times \nabla_{m} H\right)+\int_{0}^{L} \frac{\delta H}{\delta r} \cdot\left(\nabla_{m} F \times r\right) d s+\int_{0}^{L} \frac{\delta H}{\delta M} \cdot\left(\nabla_{m} F \times M\right) d s .
$$

Comparing (3.10) and (3.11) and using the vector identity $A \cdot(B \times C)=B \cdot(C \times A)$, we get

$$
\dot{\mathrm{m}}=\boldsymbol{m} \times \nabla_{m} H+\int_{0}^{L} r \times \frac{\delta H}{\delta r} d s+\int_{0}^{L} M \times \frac{\delta H}{\delta M} d s .
$$

Similarly, by considering functions $F=F(r)$ of $r$ alone and noting that $\dot{F}=$ $\int_{0}^{L} \frac{\delta F}{\delta r} \cdot \dot{r} d s$, we get

$$
\dot{r}=\frac{\delta H}{\delta M}+r \times \nabla_{m} H .
$$

A similar calculation with $F$ dependent on $M$ alone yields

$$
\dot{M}=-\frac{\delta H}{\delta r}-\nabla_{m} H \times M .
$$

"Some prefer to call this a "string" model. This model deliberately does not include second derivatives of $r$ in the energy expression (i.e. fourth derivative in the equation), since the present model is more appropriate for eneralizations to Kirchofi-Love type rod models to be considered in another publication. See MaGraB [1979], Chapters 4, S, ANmun [1974], ANTman \& KenNY [1981], and Simo [1985] and references therein for more information. 
The functional derivatives in (3.12)-(3.14) can be computed using the definition of functional derivative:

$$
\int_{0}^{1} \frac{\delta f}{\delta \phi} \cdot \delta \phi d s=\lim _{\varepsilon \rightarrow 0} \frac{f(\phi+\varepsilon \delta \phi)-f(\phi)}{\varepsilon} .
$$

In the present context, we compute

$$
\begin{gathered}
\nabla_{m} H=J^{-1} m \quad \text { where } J=\operatorname{diag}\left(I_{1}, I_{2}, I_{3}\right), \\
\frac{\delta H}{\delta r}=-A \frac{\partial^{2} r}{\partial s^{2}},
\end{gathered}
$$

and

$$
\frac{\delta H}{\delta M}=\frac{M}{\varrho_{0}} .
$$

Substituting these into (3.12)-(3.14) and, noting that $M \times M=0$, we get the equations of motion of our system:

$$
\begin{gathered}
\dot{m}=m \times J^{-1} m-\int_{0}^{2} r \times A \frac{\hat{c}^{2} r}{\partial s^{2}} d s, \\
\dot{r}=\frac{M}{\varrho_{0}}+r \times J^{-1} m, \\
\dot{M}=A \frac{\partial^{2} r}{\partial s^{2}}+M \times J^{-1} m .
\end{gathered}
$$

Notice that

$$
r \times A \frac{\partial^{2} r}{\partial s^{2}}=\frac{\partial}{\partial s}\left(r \times A \frac{\partial r}{\partial s}\right)-\frac{\partial r}{\partial s} \times A \frac{\partial r}{\partial s}
$$

and so, using the boundary conditions,

$$
\int_{0}^{L} \frac{\partial}{\partial s}\left(r \times \frac{\partial r}{\partial s}\right) d s=r \times\left.\frac{\partial r}{\partial s}\right|_{0} ^{L}=r(L) \times j-a j \times\left(\frac{\partial r}{\partial s}\right)_{s=0} .
$$

Hence the system of equations of motion (3.18) simplifies to take the final form:

$$
\begin{gathered}
\dot{m}=m \times J^{-1} m+a j \times A\left(\frac{\partial r}{\partial s}\right)_{s=0}-r(L) \times j+\int_{0}^{L} \frac{\partial r}{\partial s} \times A \frac{\partial r}{\partial s} d s, \\
\dot{r}=\frac{M}{\ell_{0}}+r \times J^{-1} m, \\
\dot{M}=A \frac{\hat{c}^{2} r}{\partial s^{2}}+M \times J-1 m .
\end{gathered}
$$


The system (3.18') has smooth solutions globally defined for all $t \geqq 0$. This can be proved using routine methods of nonlinear analysis and the a priori esti-. mate provided by conservation of energy (see for example, Holsus \& MarsDEN [1978] or MARSDEN \& Hughes [1983] for similar examples). We just state the result and make a few comments.

3.1 Proposition. The initial boundary value problem (3.18'), (3.8) admits globally defined unique solutions for initial datam in $\mathbf{R}^{3}, \boldsymbol{r}$ in $H^{1}\left([0, L], \mathbf{R}^{3}\right), M$ in $H^{\circ}\left([0, L), \mathbf{R}^{3}\right)$ and satisfying (3.8). for all $t \geqq 0$. If $r$ and $M$ are $C^{\infty}$ at $t=0$, and satisfy the necessary compatibility conditions, they are $C^{\infty}$ for all time jointly in $s$ and $t$.

Remarks. One writes $\left(3.18^{\prime}\right)$ in the form $\dot{u}=\mathscr{A} u+\mathscr{B}(u)$ where $u=(m, r, M)$ $\in \mathrm{R}^{3} \times H^{1} \times H^{0}=X$ and where $\mathscr{A}$ is the linear operator

$$
\mathscr{A}\left(\begin{array}{c}
m \\
r \\
M
\end{array}\right)=\left\{\begin{array}{l}
a j \times\left. A \frac{\partial r}{\partial s}\right|_{s=0}-r(L) \times j \\
M / \varrho_{0} \\
A \partial^{2} r / C s^{2}
\end{array}\right] .
$$

Since $A$ is wave operator in the arguments $r, M$, it generates a one parameter group on $X$, with the domain of being $\mathrm{R}^{3} \times H^{2} \times H^{1}$ (with the boundary conditions imposed). The nonlinear terms $\mathscr{B}(x)$ define a $C^{\infty}$ map of $X$ to $X$, so by standard local existence theory, (3.18') generates a local flow on $X$. Because of conservation of energy, solutions remain bounded in $X$ and so are defined for all time. Finally, by theorems of regularity, the domain of any power of $\$$, namely $\mathrm{R}^{3} \times H^{s+1} \times H^{3}$, or $\mathrm{R}^{3} \times C^{\infty} \times C^{\infty}$ is invariant under the flow. (This last statement assumes the initial data satisfy the obvious necessary conditions for compatibility with a solution, such as: from $\dot{r}=\frac{M}{\varrho_{0}}+r \times J^{-1} m$ at $s=0$ we get $\left.0=\left.\frac{M}{Q_{0}}\right|_{s=0, r=0}+a j \times\left. J^{-1} m\right|_{s-0}\right)$.

Next we turn to the stationary, or equilibrium solutions of (3.18') (also called relarine equilibria of the system before reduction - see ARNOLD [1978] and MARSDEN \& Weinstein [1974]). These equilibria are given by setting $\dot{m}=0, \dot{r}=0$ and $\dot{M}=\mathbf{0}$ in (3.18'). We look for equilibria of the form

$$
m^{e}=\Omega_{e} I_{1} i, \quad r(s)=r_{2}^{(s) j,} \quad M^{e}(s)=M_{j}^{e}(s) k .
$$

where $\Omega_{e}>0$ is a constant angular velocity. We also assume that the matrix $A$ is diagonal in the body fixed frame $i, j$, $k$; i.e., that $A=\operatorname{diag}\left(k_{x}, k_{y}, k_{z}\right)$. The equilibrium conditions from the second and third equation in $\left(3.18^{\prime}\right)$ are

$$
\frac{M_{3}}{e_{0}}=r_{2} \Omega_{e}
$$


and

$$
k_{y} \frac{d^{2} r_{2}^{f}}{d s^{2}}=-M_{j} \Omega_{e}
$$

Substituting (3.20) into (3.21), we get

$$
k_{y} \frac{d^{2} r_{2}^{p}}{d s^{2}}=-r_{2}^{e} \rho_{0} \Omega_{e}^{2} .
$$

For the equation (3.22) with boundary conditions (3.8), there are two cases:

$$
\text { (i) } \cos \left(\sqrt{\frac{\varrho_{0}}{k_{y}}} \Omega_{c} L\right) \neq 0
$$

and

(ii) $\cos \left(\sqrt{\frac{\varrho_{0}}{k_{y}}} \Omega_{c} L\right)=0 \quad$ (i.e. $\left.\sqrt{\frac{\varrho_{0}}{k_{y}}} \Omega_{c} L=\frac{n \pi}{2}, n=1,3, \ldots\right)$.

If case (i) holds, then the solution of (3.22) satisfying the boundary conditions is given by

$$
r_{2}(s)=\frac{1}{\cos \left(\omega_{e} L\right)}\left\{a \cos \left[\omega_{e}(s-L)\right]+\frac{1}{\omega_{e}} \sin \left[\omega_{e} s\right]\right\}
$$

where $\omega_{e}=\sqrt{\frac{\varrho_{0}}{k_{y}}} \Omega_{e}$ and

$$
M_{3}(s)=e_{0} \Omega_{r} r_{2}(s) .
$$

If case (ii) holds, then the boundary conditions are consistent only if

$$
r_{2}(s)=a \cos \omega_{s}+\beta \sin \omega_{s} s,
$$

where $\beta$ is a free parameter. We now have a specific equilibrium point whose stability we wish to investigate.

\section{The Energy-Casimir Method}

The "Energy-Casimir method" (see Holm, Marsden, Rattu \& Wennstenn [1984, 1985]) is based on a systematic development of an original idea of ARNOLD in the context of Lie-Poisson systems and their variants. The method provides an algorithm for determining sufficient conditions for the nonlinear stability of equilibria for systems whose underlying Hamiltonian structure has a rich collection of Casimir functions. In the present context, we shall use this method to investigate the stability of the equilibrium $\left(m^{\prime}, r^{*}, M^{*}\right)$ given by (3.19) and (3.23)(3.25).

We recall now the main steps of the Energy-Casimir method. We present it in more generality than is actually needed for our example so the reader can better judge its range of applicability as well as its limitations. 
The Stability Algorithm

A. Equations of Motion and Hamiltonian. Choose a (Banach) space $P$ of fields $u$ and write the equations of motion in first order form as

$$
\dot{u}=\mathcal{F}(u)
$$

for a (nonlinear) operator $F$ mapping a domain in $P$ to $P$. Find a conserved function $H$ for (4.1); that is, a map $H: P \rightarrow \mathbf{R}$ such that $\frac{d}{d t} H(u)=0$ for any $C^{1}$ solution $u$ of (4.1). (Usually, $H$ is the energy of the system.)

Remark $A$. Often $P$ is a Poisson manifold, i.e., a manifold admitting a Poisson bracket operation $\{$,$\} on the space of real-valued functions on P$ which makes them into a Lie algebra and which is a derivation in each variable. The brackets are usually derived by reduction, as in Section 2. The equations (4.1) to which the method applies are often Hamiltonian for such a bracket structure:

$$
\dot{\boldsymbol{F}}=\{\boldsymbol{F}, \boldsymbol{H}\}
$$

where $H$ is the energy, $F$ is any function of $u \in P$, and $\dot{F}$ is its time derivative through the dependence of $u$ on $t$. Thus, in our case, this first step has already been completed.

B. Constents of Motion. Find a family of constants of the motion for (4.1). That is, find a collection of functions $C$ on $P$ such that $\frac{d}{d t} C(u)=0$ for any' $C^{1}$ solution $u$ of (4.1).

Remark B. Unless a sufficiently large family of constants of motion is found, the ensuing step (C) may not be possible. A good way to find such functions is to use the Hamiltonian formalism in Remark $A$ to find $F$ 's suh that $\{F, H\}=0$ and to find Casimir functions for the Poisson structure; that is $C$ 's such that $\{C, G\}=0$ for all $G$. In our case, a family of Casimirs is given by (3.6).

C. First Variation. Relate an equilibrium solution $u_{e}$ of (4.1) i.e., $F\left(u_{e}\right)=0$ (so that $\frac{d}{d t} u_{e}=0$ ) and a constant of the motion $C$ by requiring that $H_{C}:=H+C$ has a critical point at $u_{e}$. Note: $C$ may or may not be uniquely determined at this stage. Keep $C$ as general as possible; any freedom may be useful in step (D).

Remark C. If Remarks A and B are followed, then, in principle, such a $C$ exists, at least locally, for most equilibria. Indeed, level sets of the C's define the "symplectic leaves" of the Poisson structure $\{$,$\} and equilibrium solutions are$ critical points of $\boldsymbol{H}$ restricted to such leaves. Thus, by the Lagrange multiplier theorem, $H+C$ has a critical point at $u_{e}$ for an appropriate Casimir function $C$. (Because of technical problems, one cannot guarantee that Casimir functions can be explicitly found in all cases.) We have yet to carry out step $C$ for our example. 
D. Convexity Estimates. Find quadratic forms $Q_{1}$ and $Q_{2}$ such that

$$
Q_{1}(\Delta u) \leqq H\left(u_{e}+\Delta u\right)-H\left(u_{e}\right)-D H\left(u_{e}\right) \cdot \Delta u
$$

and

$$
Q_{2}(\Delta u) \leqq C\left(u_{e}+\Delta u\right)-C\left(u_{e}\right)-D C\left(u_{e}\right) \cdot \Delta u
$$

for all $\Delta u$ in $P$. Require that

$$
Q_{1}(\Delta u)+Q_{2}(\Delta u)>0
$$

for all $\Delta u$ in $P, \Delta u \neq 0$.

Remark D. Formal Stability-Second Variation. As a prelude to checking (4.3), (4.4), and (4.5) it is often convenient to see whether the second variation $D^{2} H_{C}\left(u_{e}\right) \cdot(\Delta u)^{2}$, is definite, or when feasible, whether $D^{2} H_{C}\left(u_{e}\right)$ restricted to the symplectic leaf through $u_{e}$ is definite. This is a prerequisite for step (D) to work, but it is not always sufficient (see also Remark 2 below).

E. A Priori Estimates. If steps (A) to (D) have been carried out, then for any' solution $u$ of (4.1) we have the following estimate on $\Delta u=u-u_{e}$ :

$$
Q_{1}(\Delta u(t))+Q_{2}(\Delta u(t)) \leqq H_{C}(u(0))-H_{C}\left(u_{e}\right) .
$$

(This is proved below.)

F. Nonlinear Stability. Suppose steps (A) to (D) have been carried out. Then if we set

$$
\|v\|^{2}=Q_{1}(v)+Q_{2}(v)>0 \text { (for } v \neq 0 \text { ), }
$$

so $\|v\|$ defines a norm on $P$, and if $H_{C}$ is continuous in this norm at $u_{e}$, and provided solutions to (4.1), exist then $u_{e}$ is nonlinearly stable, i.e. for every $\varepsilon>0$ there is a $\delta>0$ such that if $\left\|u-u_{e}\right\|<\delta$ at $t=0$, then $\left\|u-u_{e}\right\|<\varepsilon$ for all $t$. (This represents dynamic stability against finite perturbations using the full nonlinear equations.) Should solutions of (4.1) not be known to exist for all time, we still have "conditional stability:" i.e. stability as long as $C^{1}$ solutions exist. A sufficient condition for continuity of $H_{C}$ is the existence of positive constants $C_{1}$ and $C_{2}$ such that

$$
\begin{aligned}
& H\left(u_{e}+\Delta u\right)-H\left(u_{e}\right)-D H\left(u_{e}\right) \cdot \Delta u \leqq C_{1}\|\Delta u\|^{2}, \\
& C\left(u_{e}+\Delta u\right)-C\left(u_{e}\right)-D C\left(u_{e}\right) \cdot \Delta u \leqq C_{2}\|\Delta u\| .
\end{aligned}
$$

In this case, one gets the stability estimate

$$
\begin{aligned}
\|\Delta u(t)\|^{2}:=Q_{1}(\Delta u(t))+ & Q_{2}(\Delta u(t)) \leqq C_{1} Q_{2}(\Delta u(0))+C_{2} Q_{2}(\Delta u(0)) \\
& \leqq\left(C_{1}+C_{2}\right)\|\Delta u(0)\|^{2} .
\end{aligned}
$$

(These assertions are proved below.) 
Proof of a priori extimate (4.0). Adding (4.8) and (4.9) gives

$$
\begin{aligned}
Q_{1}(\Delta u)+Q_{2}(\Delta u) & \leqq H_{C}\left(u_{e}+\Delta u\right)-H_{C}\left(u_{e}\right)-D H_{C}\left(u_{e}\right) \cdot \Delta u \\
& =H_{C}\left(u_{e}+\Delta u\right)-H_{C}\left(u_{e}\right)
\end{aligned}
$$

since $D H_{C}\left(u_{c}\right)=0$ by step (C). Because $H_{C}$ is a constant of the motion, $H_{C}\left(u_{e}+\Delta u\right)-H_{C}\left(u_{e}\right)$ equals its value at $t=0$, which is (4.6).

Proof of the Assertion in Step (F). We prove nonlinear (Liapunov) stability of $u_{e}$ as follows. Given $\varepsilon>0$, find a $\delta$ such that $\left\|u-u_{e}\right\|<\delta$ implies $\left|H_{C}(u)-H_{C}\left(u_{e}\right)\right|<\varepsilon$. Thus, if $\left\|u(0)-u_{e}\right\|<\delta$, then (4.6) gives

$$
\left\|u(t)-u_{e}\right\| \leqq\left|H_{C}(u(0))-H_{c}\left(u_{c}\right)\right|<\varepsilon .
$$

Thus, $u(t)$ never leaves the $\varepsilon$-ball about $u_{e}$ if it starts in the $\delta$-ball, so $u_{e}$ is nonlinearly stable. To see that (4.8) and (4.9) suffice for continuity of $H_{C}$ at $u_{e}$, add them to give, as in the proof of (4.6),

$$
\left|H_{C}\left(u_{e}+\Delta u\right)-H_{C}\left(u_{e}\right)\right| \leqq\left(C_{1}+C_{2}\right)\|\Delta u\|^{2}
$$

which implies that $H_{C}$ is continuous at $u_{e}$.

Remarks. 1. In many examples, including ours, $Q_{1}$ and $Q_{2}$ are each positive (so $H$ and $C$ are individually convex). Then (4.5) is automatic. However, as already noted by ARNOLD [1966b] (see also HoLM, MARSDEN \& RATU [1985]), there are some interesting examples where $Q_{1}$ is positive, $Q_{2}$ is negative, and yet $Q_{2}$ "beats" $Q_{2}$ and (4.5) is valid. If $Q_{2}$ "beats" $Q_{1}$ so $Q_{1}+Q_{2}$ is negative, then one can apply analogous procedures with $H+C$ replaced by $-(H+C)$.

2. In some cases, it is sufficient to check formal stability, i.e.; definiteness of the second variation of $H_{C}$ at $u_{e}$. This is the case for all finite dimensional examples and also often occurs when the fields are functions of a single spatial variable, such as in the KdV equation (BeNJAMan [1972] and BoNA [1975]). This remark is based on the use of Sobolev inequalities special to one dimension. In our example, we shall use a combination of convexity and Sobolev estimates. In other examples, such as two or three dimensional nonlinear elasticity it is known that definiteness of the second variation is not sufficient (see BALL \& MARSDEN [1984]).

3. In examples where solutions form shocks, the solutions leave the space $P$ and the stability algorithm may apply only up to the first shock time. Shocks may form, for example, in flows of compressible fluids; see HoLm and others [1983], [1985], for discussions of conditional stability for such cases. This is not an issue in our example since, by Proposition 3.1, solutions are smooth and exist globally in time.

4. More delicate analytic techniques than those employed in the examples here are sometimes needed to obtain or play the role of the convexity estimates. This occurs in the stability of the circular vortex patch in two dimensional flows of incompressible fluids that was proved by WaN \& PULVIRENTE [1985].

5. As already noted, in systems with a finite number of degrees of freedom, formal stability implies nonlinear stability. This fact was used by ARNOLD [1966a] 
to reproduce the well known results on stability of rigid body motion (see also Holm, Marsden, Ratiu \& Wetnstein [1984]). See Marsden \& Weinstein [1974] for the relations of the ideas on formal stability to the stability of relative equilibria and reduction.

6. For Hamiltonian systems with additional symmetries, there will be additional constants of the motion besides Casimir functions. These are usually incorporated into the expression for $C$ in step $B$. This is needed in fluid examples with a translational symmetry, for example, and in the stability analysis of a heavy top. (See Holm, Marsden, RatuU \& Weinstein [1984].)

7. For two dimensional flows of incompressible fluids, the appropriate Casimir function is the generalized enstrophy. This suggests, as is mentioned in BRETHERTON \& HAIDVOGEL [1976] and LeIH [1980], that the Casimir functions may play a role in the "selective decay hypotheses" when dissipation is added. As has been noted by MORRIsON [1985], Casimirs can be identified with the entropy functions for many systems. A similar situation occurs in spacecraft dynamics with internal rotors, and damping, as is discussed in KRISHNAPRASAD [1985]; we plan to deal with this aspect in another publication.

\section{Stability of Equilibria for Rigid Body Motion with a Flexible Attachment}

We now apply the Energy-Casimir method to determine the stability of the equilibrium solution of $\left(3.18^{\prime}\right)$ given by (3.19), (3.23) and (3.24). We have already completed steps $A$ and $B$ and so now turn to step $C$.

Taking $C$, to be of the form

$$
C_{\phi}=\frac{1}{2} \phi\left(\left\|m+\int_{0}^{L} r \times M d s\right\|^{2}\right)
$$

and letting $\alpha=0_{2} I_{1}+\int_{0}^{L} r_{2} M_{3}^{r} d s$, we find that the first variation conditions in step $C$, namely $\left.\delta\left(H+C_{\diamond}\right)\right|_{\left(m^{e}, N^{e}, M^{e}\right)}=0$, reduce to

$$
\begin{gathered}
\Omega_{e}+\alpha \phi^{\prime}\left(\alpha^{2}\right)=0, \\
k_{y} \frac{d^{2} r_{2}^{\prime}}{d s^{2}}-M_{3} a \phi^{\prime}\left(\alpha^{2}\right)=0,
\end{gathered}
$$

and

$$
r_{2}^{0} \alpha \phi^{\prime}\left(\alpha^{2}\right)+\frac{M_{3}^{c}}{\varrho_{0}}=0 .
$$

As long as $\phi$ satisfies (5.1) the conditions (5.2) and (5.3) automatically follow from (3.20) and (3.21). Thus we have determined the value of $\phi^{\prime}$ at one point, i.e.,

$$
\alpha \phi^{\prime}\left(\alpha^{2}\right)=-\Omega_{e} \text {. }
$$

This is the only requirement on $\phi$ for step $C$ to hold. 
For step $D$, we begin by writing down an explicit formula for the second variation at $\left(\boldsymbol{m}^{\odot}, \boldsymbol{r}^{+}, \boldsymbol{M}^{\circ}\right)$. In fact,

$$
\begin{gathered}
\text { S.V. }:=D^{2}\left(H+C_{\phi}\right)_{\left(m^{r}, r^{e}, M^{r}\right)} \cdot(\delta m, \delta r, \delta M)^{2} \\
=\sum_{i=1}^{3} \frac{\delta m_{i}^{2}}{I_{l}}+\int_{0}^{L} \frac{\|\delta M\|^{2}}{\varrho_{0}} d s+\int_{0}^{L}<\frac{d}{d s} \delta r, A \frac{d}{d s} \delta r>d s-\frac{\|\delta \mu\|^{2} \Omega_{e}}{\alpha} \\
-2 \Omega_{e} \int_{0}^{L}(\delta r \times \delta M)_{1} d s+2 \phi^{\prime \prime}\left(\alpha^{2}\right) \alpha^{2}(\delta \mu)_{l}^{2}
\end{gathered}
$$

where $\delta \mu=\delta m+\int_{0}^{L} \delta r \times M^{e} d s+\int_{0}^{L} r \times \delta M d s$.

We can impose the condition

$$
2 \phi^{\prime \prime}\left(\alpha^{2}\right) \alpha^{2}=\frac{\Omega_{e}}{\alpha},
$$

without violating (5.4), and so

$$
\begin{aligned}
& \text { S.V. }=\frac{\delta m_{1}^{2}}{I_{1}} \div \delta m_{2}^{2}\left(\frac{1}{I_{2}}-\frac{\Omega_{f}}{\alpha}\right) \div \delta m_{3}^{2}\left(\frac{1}{I_{3}}-\frac{\Omega_{f}}{\alpha}\right)-\frac{\Omega_{f}}{\alpha}\left[\left(\int_{0}^{L} \delta r_{1} M_{3} d s\right)^{2}\right. \\
& \left.+\left(\int_{0}^{L} \delta M_{1} r_{2} d s\right)^{2}\right]+\frac{2 \Omega_{e}}{\alpha} \delta m_{2} \int_{0}^{L} \delta r_{1} M_{3} d s+\frac{2 \Omega_{e}}{\alpha} \delta m_{3} \int_{0}^{L} \delta M_{1} r_{2} d s \\
& -2 \Omega_{e} \int_{0}^{L} \delta r_{2} \delta M_{3} d s+2 \Omega_{e} \int_{0}^{L} \delta r_{3} \delta M_{2} d s+\int_{0}^{L} \frac{\|\delta M\|^{2}}{\varrho_{0}}+\int_{0}^{L}<\frac{d}{d s} \delta r, A \frac{d}{d s} \delta r>d s \\
& =\frac{\delta m_{1}^{2}}{I_{1}}+\left[\delta m_{2}^{2}\left(\frac{1}{I_{2}}-\frac{\Omega_{e}}{a}\right)+\frac{2 \Omega_{e}}{a} \delta m_{2} \int_{0}^{L} \delta r_{1} M_{3}^{f} d s+\beta_{1}\left(\int_{0}^{L} \delta r_{1} M_{3}^{f} d s\right)^{2}\right] \\
& +\left[\delta m_{3}^{2}\left(\frac{1}{I_{3}}-\frac{\Omega_{e}}{\alpha}\right)+\frac{2 \Omega_{e}}{\alpha} \delta m_{1}\left(\int_{0}^{L} \delta M_{1} r_{2} d s\right)+\beta_{2}\left(\int_{0}^{L} \delta M_{1} r_{2}^{r} d s\right)^{2}\right] \\
& +\left(\int_{0}^{L} \delta r_{1} M_{3}^{e} d s\right)^{2}\left[-\frac{\Omega_{e}}{a}-\beta_{1}\right]+\left(\int_{0}^{L} \delta M_{1} r_{2}^{e} d s\right)^{2}\left[-\frac{\Omega_{e}}{\alpha}-\beta_{2}\right] \\
& +\int_{0}^{L} \frac{\| \delta M^{2}}{\varrho_{0}} d s+\int_{0}^{L}\left\|\frac{d}{d s} \delta r\right\|^{2} d s-2 \Omega_{e} \int_{0}^{L} \delta r_{2} \delta M_{3} d s+2 \Omega_{e} \int_{0}^{L} \delta r_{3} \delta M_{2} d s
\end{aligned}
$$


where

$$
\beta_{1}=\frac{\Omega_{e}^{2} I_{2}}{\alpha\left(\alpha-\Omega_{e} I_{2}\right)}, \quad \beta_{2}=\frac{Q_{e}^{2} I_{3}}{\alpha\left(\alpha-\Omega_{e} I_{3}\right)}
$$

Recall that

$$
\alpha=\Omega_{c} I_{1}+\int_{0}^{L} \xi_{2} M_{3} d s
$$

If we require that

$$
\alpha-J_{2} \Omega_{e}=\left(I_{1}-I_{2}\right) \Omega_{e}+\int_{0}^{L} \varrho_{0} \Omega_{e}\left(I_{2}\right)^{2} d s>0
$$

(which holds if $\mathscr{I}_{1}>I_{2}$, where $I_{1}=I_{1}+\int_{0}^{L} e_{0}\left(F_{2}\right)^{2} d s$ ) and

$$
\alpha-I_{3} \Omega_{e}=\left(I_{1}-I_{3}\right) \Omega_{e}+\int_{0}^{L} e_{0} \Omega_{e}\left(I_{2}\right)^{2} d s>0
$$

(which holds if $\boldsymbol{S}_{1}>I_{3}$ ) then use of the Cauchy-Schwarz inequality shows that the terms in the first two block parentheses in (5.8) become bounded below by perfect squares. The following Poincaré-type inequality holds, (see also HoLM, Marsden \& RatuU [1985]):

$$
\int_{0}^{2}\left(\frac{d f}{d s}\right)^{2} d s \geqq C \int_{0}^{L} f^{2} d s
$$

where $C=\pi^{2} / 4 L^{2}$ is the lowest eigenvalue of $-d^{2} / d s^{2}$ on $[0, L]$ with the homogeneous boundary conditions $f(0)=0, f^{\prime}(L)=0$ appropriate for $\delta$ r. (This readily follows by expanding $f$ in eigenfunctions of $\left.-d^{2} / d s^{2}\right)$. Using (5.11) and the CauchySchwarz inequality twice, we get

$$
\begin{gathered}
\text { S.V. } \geqq\left\{\frac{\delta m_{1}^{2}}{I_{1}}+[\text { [square] }+ \text { [square] }\}+\right. \\
\int_{0}^{2}\left[\delta M_{1}^{2}\left(\frac{1}{\varrho_{0}}-\gamma_{1}\right)+\delta M_{2}^{2}\left(\frac{1}{\varrho_{0}}\right)+\delta M_{3}^{2}\left(\frac{1}{\varrho_{0}}\right)+\delta r_{1}^{2}\left(k_{x} C-\gamma_{2}\right)+\delta r_{2}^{2} \cdot k_{y} C\right. \\
\left.+\delta r_{3}^{2} \cdot k_{2} C-2 \Omega_{e} \delta r_{2} \delta M_{3}+2 \Omega_{e} \delta r_{3} \delta M_{2}\right] d s
\end{gathered}
$$

where

$$
\gamma_{1}=\left(\int_{0}^{L}\left(r_{2}\right)^{2} d s\right) \frac{\Omega_{e}}{\alpha-\Omega_{e} I_{3}}, \quad \gamma_{2}=\left(\int_{0}^{L}\left(M_{3}^{\prime}\right)^{2} d s\right) \frac{\Omega_{c}}{\alpha-\Omega_{I_{2}}} .
$$

It follows that S.V. $>0$ if the integrand in (5.12) is positive.

From Sylvester's theorem, the following conditions ensure that the integrand 
in (5.12) will be positive:

$$
\begin{aligned}
& \frac{1}{\varrho_{0}}>\gamma_{1} \\
& k_{x} C>\gamma_{2} \\
& \frac{k_{y}}{\varrho_{0}} C>\Omega_{e}^{2}, \\
& \frac{k_{z} C}{\varrho_{0}}>\Omega_{e}^{2} .
\end{aligned}
$$

These inequalities together with the inequalities (5.10) ensure that S.V. is positive and hence that the considered equilibrium $\left(m^{e}, r, M^{e}\right)$ is formally stable.

We are now ready to formulate our stability criterion:

5.1 Theorem. The dynamics (3.18') of the rigid body with elastic attachment governed by the Hamilionian $H$ of equation (3.7) has a (relative) equilibrium at

$$
\begin{gathered}
m^{e}=\Omega_{e} J_{1} i \\
r=r_{2}^{e}(s) j=\frac{1}{\cos \left(\omega_{e} L\right)}\left\{a \cos \left[\omega_{e}(s-L)\right]+\frac{1}{\omega_{e}} \sin \left[\omega_{e} s\right]\right\}
\end{gathered}
$$

(where $\left.\omega_{c}=\sqrt{\varrho_{0} / k_{y}} \Omega_{c}\right)$, and

$$
M^{e}=e_{0} \Omega_{r} r_{2}^{*} k \text {. }
$$

Furthermore, in terms of $S_{1}=I_{1}+\int_{0}^{L} e_{0}\left(r_{2}\right)^{2} d s$, the effective moment of inertia, the following inegualities are sufficient conditions for the equilibrium $\left(\boldsymbol{m}^{e}, \boldsymbol{r}^{\circ}, \boldsymbol{M}^{*}\right)$ to be a nonlinearly stable equilibrium in the $\mathrm{R}^{3} \times H^{1} \times L^{2}$ norm on $(m, r, M)$ space:

$$
\begin{gathered}
I_{1}-I_{3}>0 \\
I_{1}-I_{2}>\left[\int_{0}^{L} \varrho_{0}\left(r_{2}\right)^{2} d s\right]\left(\frac{\Omega_{e}}{\sqrt{\frac{k_{x}}{\varrho_{0}} \frac{\pi}{2 L}}}\right)^{2} \\
\Omega_{e}<\sqrt{\frac{k}{\varrho_{0}}} \cdot \frac{\pi}{2 L}
\end{gathered}
$$

(c)

where $k=\min \left(k_{y}, k_{z}\right)$.

Note that (5.15)(a) (b) are similar to the rigid body stability criteria $I_{1}-J_{3}>0$, $I_{1}-I_{2}>0$ and (c) states that the angular frequency of the body should not exceed the characteristic transverse beam frequencies.

Let us now complete the proof of the theorem. The conditions (5.15) together with our preceding calculations show formal stability. To establish the precise 
estimates, in (4.3) let $Q_{1}$ be $H$ itself (since $H$ is quadratic). Let $\phi^{\prime \prime}$ be bounded below by $\frac{\Omega_{e}}{2 \alpha^{3}}$ (see (5.6). Then

$$
\begin{gathered}
C_{\phi}(m, r, M)-C_{\phi}\left(m^{e}, \mu^{e}, M^{e}\right)-D C_{\phi}\left(m^{e}, r_{,}, M^{\prime}\right) \cdot(\Delta m, \Delta r, \Delta M) \\
=\frac{1}{2}\left[\phi\left(|\mu|^{2}\right)-\phi\left(\left|\mu_{e}\right|^{2}\right)-\phi^{\prime}\left(\left|\mu_{e}\right|^{2}\right) \cdot 2 \mu_{e} \cdot\left(\Delta m+\int_{0}^{2} \rho \times \Delta M d s+\int_{0}^{2} \Delta r \times M^{e} d s\right)\right] \\
=\frac{1}{2}\left[\phi\left(|\mu|^{2}\right)-\phi\left(\left|\mu_{e}\right|^{2}\right)-\phi^{1}\left(\left|\mu_{e}\right|^{2}\right) \cdot\left(|\mu|^{2}-\mid \mu_{e}^{2}\right)+\frac{1}{2} \phi^{\prime}\left(\left|\mu_{e}\right|^{2}\right) \mu_{e} \cdot \int \Delta r \times \Delta M d s\right] \\
\geqq \frac{\Omega_{e}}{\alpha^{3}}\left(|\mu|^{2}-\left|\mu_{e}\right|^{2}\right)^{2}-\frac{1}{2} \frac{\Omega_{e}}{\alpha} \mu_{e} \cdot \int \Delta r \times \Delta M d s .
\end{gathered}
$$

For $\Delta \mu=\mu-\mu_{e}$ sufficiently small, this is

$$
\geqq \frac{\Omega_{e}}{2 \alpha^{3}}\left(\mu_{e} \cdot \Delta \mu\right)^{2}-\frac{1}{2 \alpha I_{2}} \mu_{e} \cdot \int \Delta r \times \Delta M d s=: Q_{2}(\Delta m, \Delta r, \Delta M) .
$$

The argument given for the second variation shows that (4.5) holds. The norm determined by $Q_{1}+Q_{2}$ is equivalent to the standard norm on $\mathrm{R}^{3} \times H^{1} \times L^{2}$ (by using arguments standard in elliptic theory, for example). Notice that smallness in $\mathrm{R}^{3} \times H^{3} \times L^{2}$ implies $\Delta \mu$ is small, so our disposing of these terms in (5.18) is justified. Finally. (4.8) is obvious and (4.9) holds (for $\Delta \mu$ sufficiently small) by the argument used to derive $Q_{2}$. The arguments at the end of Section 4 complete the proof.

Acknowledgments. KRushNaprusad's work was given partial support by the National Science Foundation under Grant ECS-82-19123, by the Minta Martin Fund for Aeronautical Research, and by the Office of Naval Research under Contract N00014-K-2008; MARSDEN's by DOE Contract DE-ASO3-82ER12097.

We thank Gloria alvarez-Sanchez, Daryl Hols, TOM Hughes, Tudor Ratiu, and JUAN Simo for helpful remarks.

\section{References}

R. Agrabam \& J. Marsden [1978]. Foundations of Mechanics, Second Edition, AddisonWesley.

G. Alvarez-Sanchez [1986]. Control of Hamiltonian systems with symmetry, Thesis, Univ. of Calif., Berkeley.

S. Antman [1972]. The theory of rods, Handbuch der Physik VI, C. Truesdell, ed., Springer-Verlag, 641-703.

S. ANTMAN [1974]. Kirchhoff's problem for nonlinearly elastic rods, Quart. J. Appl. Marh. 32, 221-240.

S. ANTMAN \& J. KENNY [1981]. Large buckled states of nonlinearly elastic rods under torsion, thrust and gravity, Arch. Rational Mech. An. 76, 289-354.

S. Antman \& A. Nachman [1980]. Large buckled states of rotating rods, Nontinear Analysis TMA 4, 303-327. 
V. Arwold [1966a]. Sur la gtometrie differentielle des groupes de Lie de dimeasion infinie et ses applications à l'hydrodynamique des fluids parfaits, Ann. Inst. Fowrier 16, 319-361.

V. ArNord [1966b]. An a priori estimate in the theory of hydrodynamic stability, Izv. Vyssh. Uchebn. Zved. Mat. 54, 3-5, English Transl.: Am. Math. Soc. Transl, 1979 [1969], 267-269.

V. ARNOLD [1978]. Mathematical Methods of Classical Mechanics, Springer-Verlag.

J. BALL \& J. MARSDEN [1984]. Quasiconvexity at the boundary, positivity of the socond variation and elastic stability. Arch. Rational Mech. An. 86, 251-277.

J. BNLLIEUL \& M. LEV [1983]. Dynamics of rotating texible structures, Proc. IEEE Conf. CDC, San Antonio. TP2, 808-813.

J. Ballueur [1983]. Modeling and control of flexible and articulated spacecraft, Proc. CISS, Johns Hopkins University, 95-102.

T. Benjaman [1972]. The stability of solitary waves, Proc. Roy. Soc. London 328 A, 153183.

J. BonA [1975]. On the stability theory of solitary waves, Proc. Roy. Soc. London 344 A, 363-374.

F. Bretherton \& D. Hadvogel [1965]. Two-dimensional turbulence above topography, J. Fluid Mech. 78, 129-154.

v. Gurluaman \& S. Sternmerg [1984]. Symplectic Technigues in Physics, Cambridge University Press.

D. Holm, B. Kupershmidt, \& C. Levernore [1984]. Canonical maps between Poisson brackets in Eulerian and Lagrangian descriptions of continuum mechanies, Phys. Lett. 98 A, 389-395.

D. Holm, J. Marsden, \& T. RATIU [1985]. Nonlinear stability of the Kelvin-Stuart cats eye solutions, Proc. AMS-SIAM Summer Seminar, Loctures in Applied Math. AMS vol. 123, 171-186.

D. Holm, J. Marsden, T. RATUU \& A. Wenstan [1983]. Nonlinear stability conditions and a priori estimates for barotropic hydrodynamics, Physics Letters 98 A, 15-21.

D. Holm, J. MArsoen, T. RATUU \& A. Wenstan [1984]. Stability of rigid body motion using the energy-Casimir method. Cont. Math. AMS 28, 15-23.

D. HoLM, J. MArsden, T. RATtu \& A. Wenstean [1985]. Nonlinear stability of fuid and plasma equilibria, Physics Reports 123, 1-116.

P. HOLMES \& J. MARSDEN [1983]. Horseshoes and Amold difiusion for Hamiltonian systems on Lie groups. Indiana Unio. Math. J. 32, 273-310.

C. HUBERT [1981]. The attitude dynamies of dynamies explorer A, Attitude Control Analysis, RCA Astro-Electronics, AAS 81-123.

T. KANE \& D. LEvinson [1980]. Formulation of the equations of motion for complex spacecraft, J. Guidance and Control 3, No. 2, 99-112.

T. Kune, P. Luxuns \& D. LeVInson [1983]. Spacecraft Dymamics, New York: McGrawHill.

P. KRISHNApRasad \& C. BerensteIn [1984]. On the equilibria of rigid spececraft with rotors, Systems and Control Letters 4, 157-163.

P. Krushnaprasad [1983]. Lie-Poisson structures and dual-spin spacecraft, Proc. 22nd IEEE Cons, on Decision and Control, IEEE, New York, 814-824.

P. Krushnaprusad [1985]. Lie-Poisson structures, dual-spin spacecrafi and asymptotic stability, Nonlinear Aralysis: TMA vol. 9. No. 10, 1011-1035.

C. LetrH [1984]. Minimum Enstrophy Vortex, AIP Proc. 106, 159-168.

D. LEWIS, J. MARSDEN, R. MONTGOMERY \& T. RATU [1986]. The Hemiltonian structure for dynamic free boundary problems. Physica $18 \mathrm{D}, 391-404$.

P. Likns [1974]. Analytical dynamics and nonrigid spacecraft simulation, JPL Technical Report, TR 32-1593. 
E. Magrab [1979]. Vibrations of Elastic Structural Members, Sijthoff \& Nordhoff, Netherlands.

J. Marsdan \& T. J. R. Huorres [1983]. Mathematical Foundations of Elasticily, Prentice. Hall.

J. Marsden \& T. Ratu [1986]. Reduction of Poisson Manifolds, Lett. Mash. Phys. 11, $161-170$.

J. Marsden, T. Ratju \& A. Wejnstein [1984a]. Semi direct products and reduction in mechanics, Trans. Am. Math. Soc. 281, 147-177.

J. Marsden, T. RATIU \& A. Weinstein [1984b]. Reduction and Hamiltonian structures on duals of semidirect product Lie algebras, Cont. Math. AMS 28, 55-100.

J. MARSDEN \& A. WEnstein [1974]. Reduction of symplectic manifolds with symmetry, Rep. Marh. Phys. 5, 121-130.

J. Marsden, A. Weinstejn, T. Ratiu, R. Schamd \& R. G. Spencer [1983]. Hamiltonian systems with symmetry, coadjoint orbits and plasma physics, Proc. IUTAM-ISIMM Symposium on "Modern Developments in Analytical Mechanics," Torino (June 7-11, 1982), Ani della Academia delle Scienze di Torino 117, 289-340.

1. Meirovitch [1974], Bounds on the extension of antennas for stable spinning satellites, J. Spacecraft and Rockets, March, 202-204.

L. Merovtch \& J. Juang [1974]. Dynamics of a Gravity-Gradient Stabilized Flexible Spacecraft, NASA Contractor Report: NASA CR-2456.

R. MONTGOMERY, J. MARSDen \& T. RATIU [1984]. Gauged Lie-Poisson Structures, Cont. Math. AMS, 28, 101-114.

P. Morrison [1986]. A paradigm for joined Hamiltonian and dissipative systems, Physica $18 \mathrm{D}, 410-419$.

A. Nacrman [1985]. Buckling and vibration of a rotating beam (preprint).

E. REISSNER [1973]. On a one-dimensional large-displacement finite-strain beam theory, Srudies in Appl. Math. 52, 87-95.

E. Ressser [1981]. On finite deformation of space-curved beams, ZAMP 32, 734-744.

J. C. Simo [1985]. Finite strain beam formulattion: I, to appear in Comp. Meth. App.

Mech. Engg.

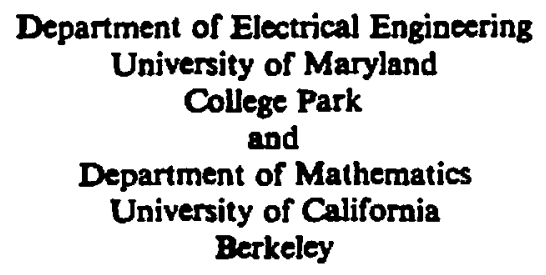

(Received December 16, 1985) 